\title{
Molecular dynamics simulations of single-layer and rotated double- layer graphene sheets under a high velocity impact by fullerene
}

\author{
Yun Qiu ${ }^{1}$, Yang Zhang ${ }^{1,2}$, A.S. Ademiloye ${ }^{2, *}$, Zhengtian $\mathrm{Wu}^{3}$ \\ ${ }^{I}$ School of Science, Nanjing University of Science and Technology, Nanjing, 210094, China \\ ${ }^{2}$ Zienkiewicz Centre for Computational Engineering, College of Engineering, \\ Swansea University Bay Campus, Swansea, SA1 8EN, UK \\ ${ }^{3}$ School of Electronic and Information Engineering, Suzhou University of Science and Technology \\ Suzhou 215009, China
}

\begin{abstract}
Molecular dynamics (MD) simulations are employed in this paper to study the behavior of single-layer and rotated double-layer graphene sheets under a high velocity impact. The AIREBO force field is used for MD simulations. Stress wave propagation is investigated, and cone-wave and axial-wave velocities are determined. The coefficient of restitution for the double-layer graphene sheet is calculated at different impact incident angles and velocities. Impact and rebound kinetic energy of projectile under the impact simulation of different rotation angles double-layer graphene sheet is monitored. High cone-wave and axial-wave velocities show that single-layer and double-layer graphene sheets have potential applications in impact protection materials.
\end{abstract}

Keywords: Molecular dynamics simulation; Double-layer graphene; Impact; Stress wave; Fullerene

*Corresponding author.

Email address: a.s.ademiloye@ @wansea.ac.uk (A.S. Ademiloye) 


\section{Introduction}

Graphene has a single layer of carbon atoms in a honeycomb configuration. It is a well-known two-dimensional atomic-scale material whose study and applications have opened up new research fields and has led to the development of various nanosensors and nanoelectromechanical systems (NEMS) [1], flexible liquid crystal displays (LCDs) [2], organic light-emitting diodes (OLEDs) and sensors (biological and chemical) [3]. Graphene has Young's modulus value of $1 \mathrm{TPa}$ and intrinsic strength of up to $130 \mathrm{GPa}$ [4]. These interesting properties have led to a wide range of potential engineering applications such as flexible electronics [5], nanoparticle mass detection [6], graphene natural rubber composites [7], coatings (corrosion resistant) [8], reinforcements (for nanocomposites) [9] and biomedical applications [10].

In order to promote various applications, great efforts have been made to achieve the mass production of graphene [11]. Through roll-to-roll technology, researchers have reported the successful production of 30-inch graphene films. Graphene sheets (GSs) have received much attention due to their exceptional mechanical, electrical properties and superior thermal conductivity $[12,13]$. Combined with its low mass density ( 2220 $\mathrm{kg} / \mathrm{m}^{3}$ ), graphene also shows attractive potential in the field of impact protection, and as a new candidate for shielding materials, such as combat armor [14], nano-reinforced composites $[15,16]$.

Graphene is naturally a good candidate for ballistic membranes because of its extraordinary strength and stiffness, measured by atomic force microscopy [17]. The specific penetration energy of multilayer graphene (MLG) membranes has been revealed through a Laser-induced Projectile Impact Test (LIPIT) experiment to be about 10 times the corresponding literature value of the macro steel plate $[18,19]$, which illustrates the potential of MLG as a bulletproof material. Natsuki et al. [20, 21] theoretically analyzed the response of double-layer graphene under transverse impact. Their results show an improved impact energy absorption in double-layer graphene due to the effect of van der Waals (vdW) forces between two layers of graphene sheets. 
In 2016, Xia et al. [22] studied the failure mechanism of monolayer graphene under hypervelocity impact of spherical projectile and observed that circular shape graphene possesses the best impact resistance for similar sample size. In an interesting study on the design of graphene-based devices, Sadegh [23] demonstrated that the impact resistance of graphene sheets is significantly influenced by the number of layers, aspect ratio, sheet size, interlayer distance, delamination, and projectile shape. Kawai et al. [24] studied the bombardments of two graphenes that are placed perpendicular to each other and show that they can be chemisorbed to form a three-dimensional Yjunction. In 2015, Bai et al. [25] systematically investigate the influence of incident angle on the generation of defects and vacancies during bombardment process, their results show that oxygen ions with the incident angle of $70^{\circ}$ produce the highest probability of ion substitution. These previous studies affirmed that the bombardment effect of fullerenes and graphene has a wide range of applications and that graphene has potential applications in impact protection materials.

This experimental paper encourages us to study rotated double-layer graphene to increase our understanding of the mechanical properties of double-layered rotating graphene under a high velocity impact.

In this paper, molecular dynamics (MD) simulations are employed to study the behavior of single-layer and rotated double-layer graphene sheets under high velocity impact by $\mathrm{C}_{180}$ fullerene projectile. Firstly, we investigate the stress wave propagation of single-layer GSs and calculated its cone-wave and axial-wave velocities. Following this, a model of a double-layer graphene sheet is developed to study the coefficient of restitution during impact. Finally, the double-layer graphene sheet with different rotation angles under the impact of fullerene projectile with the same initial kinetic energy is studied. Our numerical results show that the double-layer graphene with high rotation angle has better performance in terms of energy absorption. 


\section{Simulation method}

In this study, all the simulations are performed with the Large-scale Atomic/Molecular Massively Parallel Simulator (LAMMPS) package [26], and atom visualization is done by Visual Molecular Dynamics (VMD) [27]. The Adaptive Intermolecular Reactive Empirical Bond Order (AIREBO) [28] force field is used for all the intra- and inter-molecular interactions, and AIREBO potential has been widely used to study the mechanical properties of carbon systems, the cutoff distances are defined in the potential $\left(r_{c c}^{\min }=1.7 \AA\right.$ and $\left.r_{c c}^{\max }=2.0 \AA\right)$. Time step is taken as 0.5 fs and temperature is considered as $1 \mathrm{~K}$ in all simulations.

\subsection{Stress wave analysis of single-layer graphene sheet}

To study the stress wave propagation of single-layer graphene sheet, a large size graphene sheet with dimensions $60 \mathrm{~nm} \times 60 \mathrm{~nm}$ has been considered. The single-layer graphene sheet is impacted by a $C_{180}$ fullerene projectile with a mass of $3.58 \times 10^{-24} \mathrm{~kg}$ and a diameter of $1.2 \mathrm{~nm}$. The $\mathrm{C}_{180}$ fullerene was selected as the impactor due to its spherical shape, which helps to simplify the model and theoretical analysis. It is initially located at a distance of $2 \mathrm{~nm}$ above the center of the graphene sheet to avoid any interactions between the $\mathrm{C}_{180}$ atoms and the graphene atoms. The fullerene projectile is considered as a rigid body to prevent large deformations during high velocity impact. Before the impact simulation, the system is relaxed for $5 \mathrm{ps}$, and the impact simulation is conducted with NVE ensemble. All edges of the graphene sheet are fixed during the simulation. As shown in Fig. 1, in order to calculate cone-wave and axial-wave velocities, several points along the $x$-axis direction of the graphene were identified and marked to measure displacements. The $z$-direction $\left(u_{z}\right)$ displacement of points A0 - A5 is measured, and the $x$-direction $\left(u_{x}\right)$ displacement of points A6 - A9 is measured. By measuring the displacement of the nearest 4 atoms to the coordinate positions of points A0 - A9 and taking a statistical average, the displacement of each of these points of interest can be obtained. 


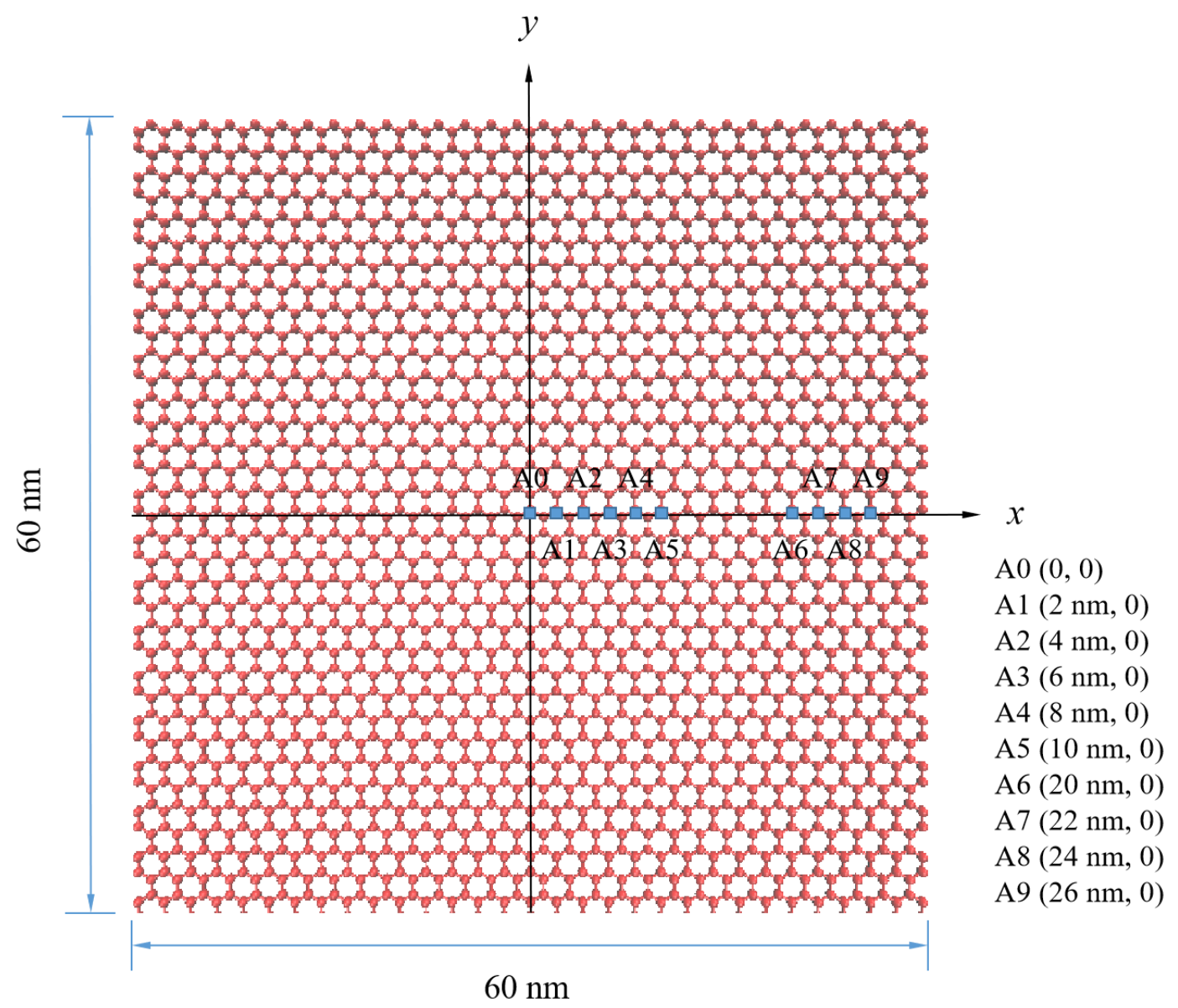

Fig. 1. Schematic illustration of a graphene sheet with a size of $60 \mathrm{~nm} \times 60 \mathrm{~nm}$ employed for stress wave calculations.

\subsection{Coefficient of restitution for double-layer graphene sheet}

In order to investigate the coefficient of restitution for double-layer graphene sheet, the simulation system consists of a $\mathrm{C}_{180}$ fullerene and a double-layer graphene sheet with dimensions $20 \mathrm{~nm} \times 20 \mathrm{~nm}$. The fullerene, $C_{180}$, is initially located above the center of the graphene sheet at a sufficiently large distance $(2 \mathrm{~nm})$ to avoid any interaction between the $\mathrm{C}_{180}$ atoms and the graphene atoms. All edges of the graphene sheet are fixed, and the fullerene projectile is considered as a rigid body during the simulation. Before the projectile is allowed to move, the graphene sheet is relaxed for $5 \mathrm{ps}$, and the impact simulation is conducted with NVE ensemble. The incident angle $(\theta)$, as shown in Fig. 2, is varied from $30^{\circ}$ to $90^{\circ}$. The energy of the fullerene projectile is varied from 
8.4 to $179.24 \mathrm{eV}$. During the simulation, the velocity of the projectile was measured and employed to calculate the coefficient of restitution along the $x$ and $z$ directions.

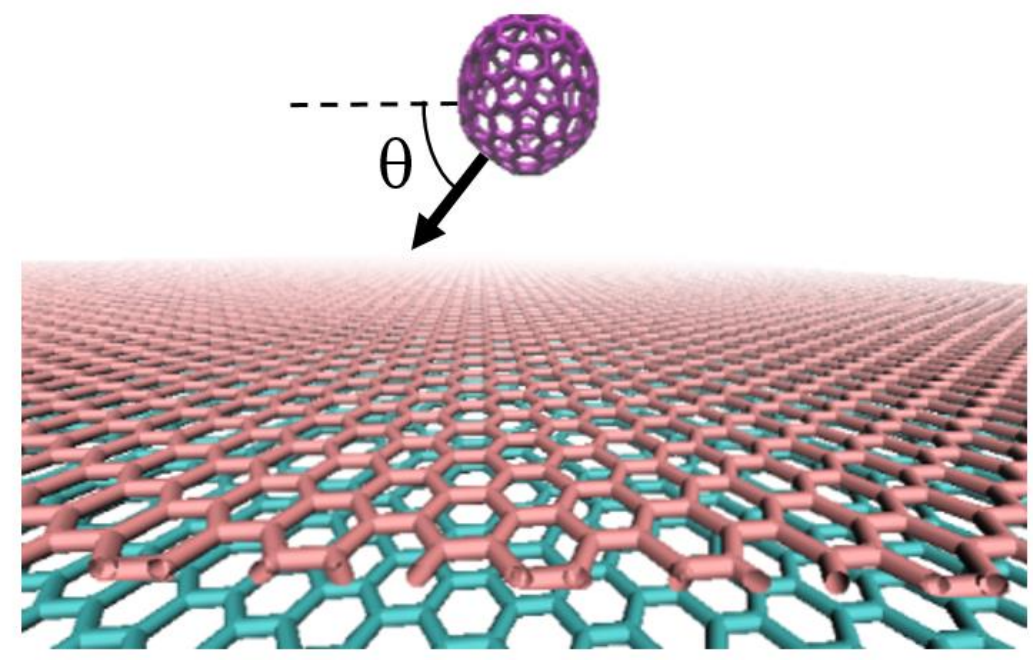

Fig. 2. Definition of the incident angle, $\theta$ and a schematic representation of its initial position relative to the double-layer graphene sheet

\subsection{Impact on a rotated double-layer graphene sheet}

To compute the impact property of a rotated double-layer graphene sheet, the model as shown in Fig. 3 was developed. Unlike the preceding cases, in this simulation, each layer of the graphene sheet is circular in shape with a diameter of $20 \mathrm{~nm}$. The distance between adjacent graphene layers is set to $0.34 \mathrm{~nm} . \mathrm{C}_{180}$ fullerene is used as the projectile in our study. It is treated as a rigid body and place at an initial distance of $2.0 \mathrm{~nm}$ from the top graphene sheet. Before the projectile is allowed to move, the graphene sheet is relaxed for 5 ps, and the impact simulation is conducted with NVE ensemble. Kim et al. [29] conducted a systematic Raman spectroscopy study on rotated double-layer graphene with angles from $0^{\circ}$ to $30^{\circ}$ with an increment of about $1^{\circ}$ and measured the intensity, peak position, and peak width of graphene 2D and G Raman modes. Their results show that the inflection points of the curve occur at several specific rotation angles $\left(3^{\circ}, 7^{\circ}, 10^{\circ}, 14^{\circ}, 20^{\circ}\right.$ and $\left.27^{\circ}\right)$. Therefore, following the findings in Refs. $[29,30]$, the rotated double-layer graphene sheet with rotation angles of $3^{\circ}, 7^{\circ}, 10^{\circ}, 14^{\circ}$, 
$20^{\circ}$ and $27^{\circ}$ is adopted in our present contribution and impacted by the $\mathrm{C}_{180}$ fullerene with the energy of $100.825 \mathrm{eV}$. The kinetic energy of the projectile was constantly monitored during the simulation.

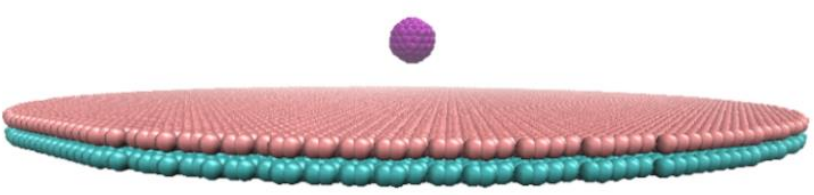

Fig. 3. Schematic of rotated double-layer graphene sheet with a diameter of $20 \mathrm{~nm}$

\section{Results and discussions}

\subsection{Stress wave analysis}

To compute the stress wave propagation, a $\mathrm{C}_{180}$ fullerene with a velocity of $4 \mathrm{~km} / \mathrm{s}$ impacts a single-layer graphene sheet with dimensions $60 \mathrm{~nm} \times 60 \mathrm{~nm}$. During the impact, the graphene sheet shows a downward deformation cone. As shown in Fig. 4, at $t=0.74 \mathrm{ps}$, the maximum displacement $\left(u_{z}=-0.551 \mathrm{~nm}\right)$ appears at the center of the graphene sheet (point A0 at $x=0$ ). The time history of $z$-direction displacement $\left(u_{z}\right)$ of points A0 - A5 can be seen in Fig. 4. For point A1 - A5, the farther away from the center, the smaller the maximum deformation displacement. When the cone-wave propagation (effect of bending wave propagation [31]) ceases, the point A1 - A5 achieve a steady downward deformation (at $u_{z}=-0.045 \mathrm{~nm}$ ). Dividing the inter-point distance with the arrival time at each point, the cone-wave velocity has been calculated to be $4.14 \mathrm{~km} / \mathrm{s}$. 


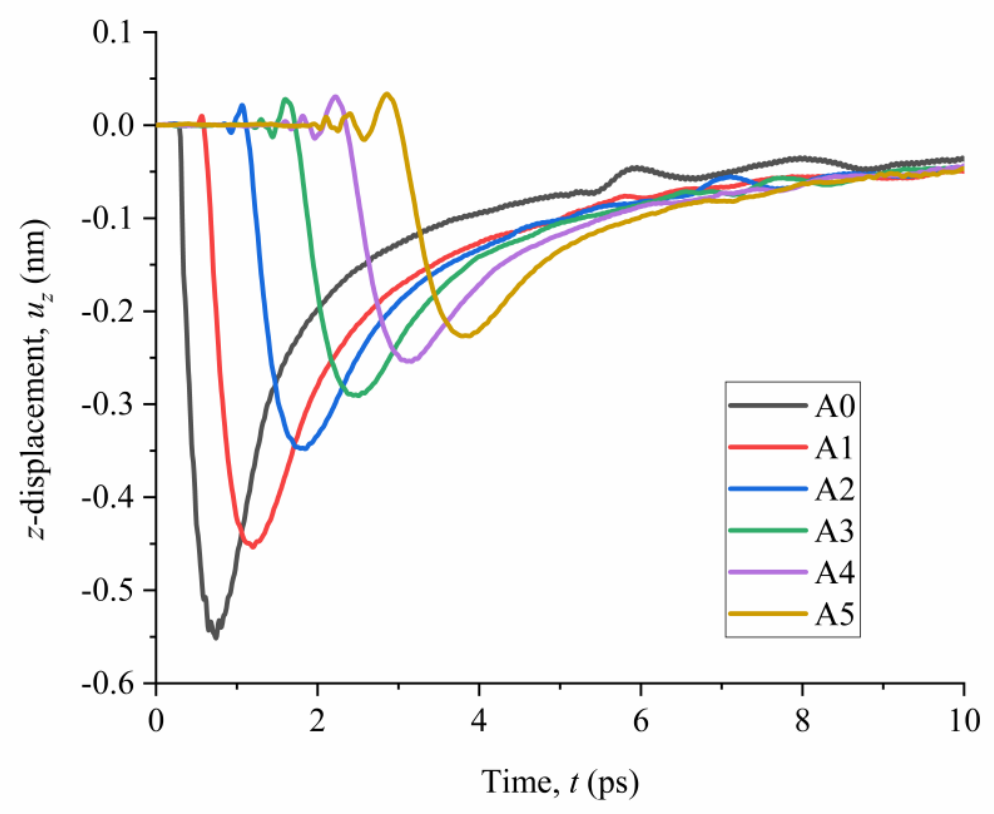

Fig. 4. Time history of $z$-displacement $\left(u_{z}\right)$ of the points A0 - A5.

Fig. 5. shows the time history of $x$-displacement of the points A6 - A9. The axialwave velocity is calculated to be $18.18 \mathrm{~km} / \mathrm{s}$ by dividing the inter-point distance $(2 \mathrm{~nm})$ with the arrival time at points.

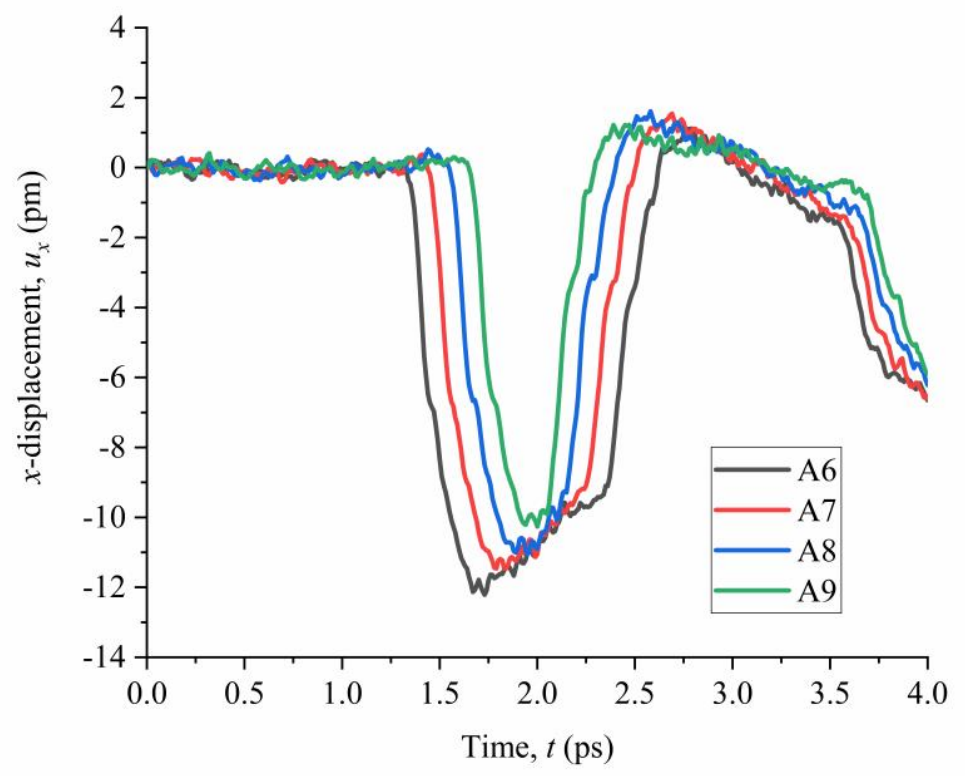

Fig. 5. Time history of $x$-displacement $\left(u_{x}\right)$ of the points A6 - A9. 


\subsection{Coefficient of restitution}

In this section, the response of double-layer graphene under transverse impact $(\theta$ $=90^{\circ}$ ) is analyzed in order to study the coefficient of restitution. The time history of displacement at the center of the graphene sheet (corresponding to the displacement of the atom directly below the place of impact) was also recorded. To avoid the perforation of graphene sheet under high velocity impact [32], the velocity of the projectile in this simulation is $3 \mathrm{~km} / \mathrm{s}$. Fig. 6 shows the displacement of the top and bottom graphenes under transverse impact. We observed that during the impact of the projectile (the contact of the projectile with the top graphene), the top graphene sheet is largely deformed, and its displacement in the $z$-direction is larger than the corresponding displacement of bottom layer graphene. The displacement in the $z$-direction begins to decrease as the projectile rebounds. At this time, the $z$-direction displacement of the top graphene sheet is smaller than that of the bottom layer graphene.

According to the analytical model presented in Ref. [20] for double-layer graphene under transverse impact, double-layer graphene sheet can be considered as two different graphene sheets bound together by van der Waals (vdW) forces. When the projectile hits the graphene sheets and they come into contact, a local contact deformation in the top layer graphene occurs. The local indentation creates a pressure distribution which causes the top graphene to be loaded globally and produce dynamic deformation, while the deformation of the bottom graphene sheet is due to the vdW forces between the top and bottom graphene sheets. The impact kinetic energy obtained from the projectile converted into the deformation energy of graphene. Therefore, during the contact of the projectile, the displacement of the top graphene sheet in the $z$-direction is greater than that in the bottom layer, and the influence of the vdW forces at this time is small.

When the projectile leaves the surface of the graphene, a repulsive vdW force is generated between the top and bottom layers, so that the displacement of the top graphene in the $z$-direction is smaller than that of the bottom graphene. Since the bottom graphene sheet has a vdW force on the top graphene, it can effectively resist 
deformation and absorb energy, and the double-layer graphene has better protection ability under impact than single-layer graphene.

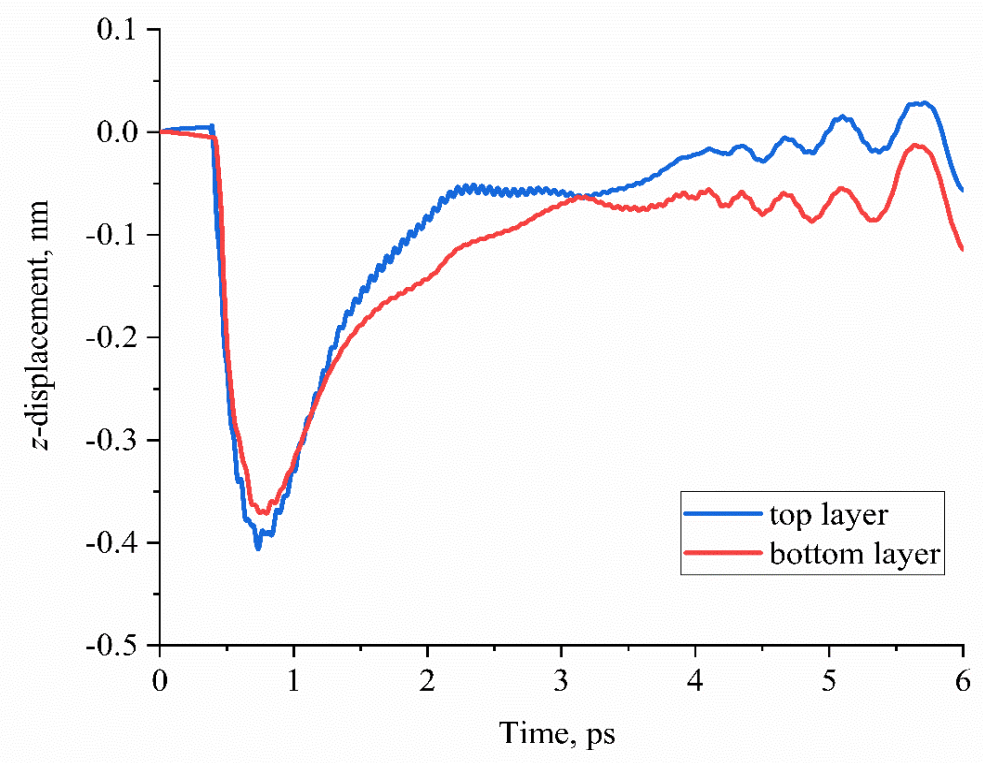

Fig. 6. Time history of displacements for top and bottom layer graphene at the center of graphene

The coefficient of restitution is an important parameter for addressing the impact of macroscopic bodies. According to its standard definition, the recovery factor is the ratio of the normal component of the rebound velocity $V_{2}$ to the impact velocity $V_{l}(R C$ $\left.=V_{2} / V_{1}\right)$ when the affected object is fixed. To prevent the perforation of the graphene sheets under high velocity impact [32], the velocity of the rigid body projectile employed in our simulations is below $4 \mathrm{~km} / \mathrm{s}$. At this impact velocity, the structure of the graphene sheet is completely preserved and without any crack or perforation. Nonrigid fullerene will stick to the graphene during low-velocity impacts while its structure will disintegrate during high-velocity impacts. The unrecoverable buckling deformation of the fullerene projectile after impact will dissipate part of the impact kinetic energy [33]. Therefore, in order to better evaluate the energy absorption effect of graphene sheets rather than fullerene projectile, we considered the fullerene 
projectile as a rigid body such that the dissipated energy is only converted to thermal energy.

In this subsection, the coefficient of restitution is used as a parameter to evaluate the energy absorption of the graphene sheet in the $x$ and $z$ directions. Impact and rebound velocities of the projectile during the impact simulation of double-layer graphene sheet were monitored. Figs. 7 and 8, respectively, show the coefficient of restitution for the fullerene projectile impact along the $x$ and $z$ directions at various impact velocities. As shown in Fig. 7, each curve represents a collision at different angles $\left(\theta=30^{\circ}, 45^{\circ}\right.$ and $\left.60^{\circ}\right)$. It can be seen from Figs. 7 and 8 that the coefficient of restitution in the vertical direction $\left(R C_{z}\right)$ is significantly smaller than the coefficient of restitution in the horizontal direction $\left(R C_{x}\right)$. The coefficient of restitution in the horizontal direction ( $x$-direction) is above 0.8 , which means that during the collision, the velocity of the projectile in the horizontal direction remains high, and the graphene sheet absorbs less energy in the horizontal direction. In the vertical direction $(z-$ direction), the coefficient of restitution is below 0.5 , and the velocity of the projectile is significantly reduced. The graphene sheet has a better energy absorption effect in the vertical direction. As the impact velocity increases, the coefficient of restitution in the vertical direction $\left(R C_{z}\right)$ and horizontal direction $\left(R C_{x}\right)$ both decrease. This may be due to greater resistance developed by the graphene sheet with an increase in impact velocity. As the angle of incidence increases, the value of $R C_{z}$ increases. Conversely, for $R C_{x}$, it decreases with the increase of incident angle. 


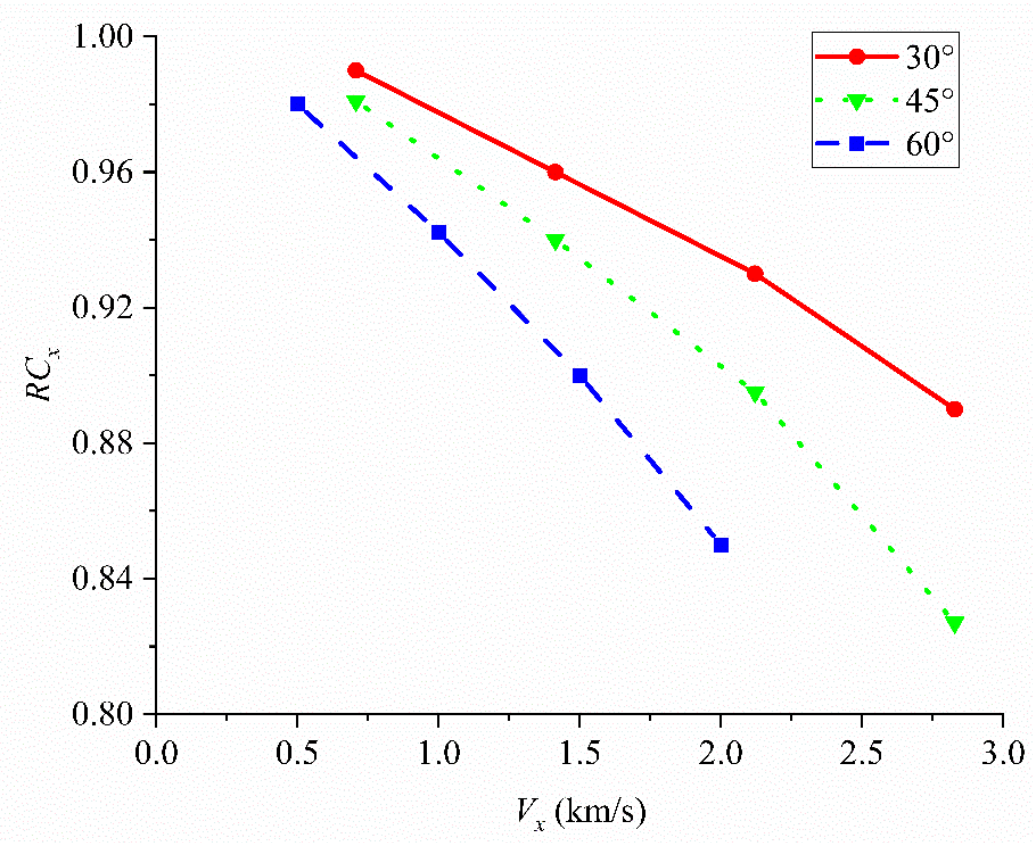

Fig. 7. Coefficient of restitution for the projectile impact along the $x$-direction.

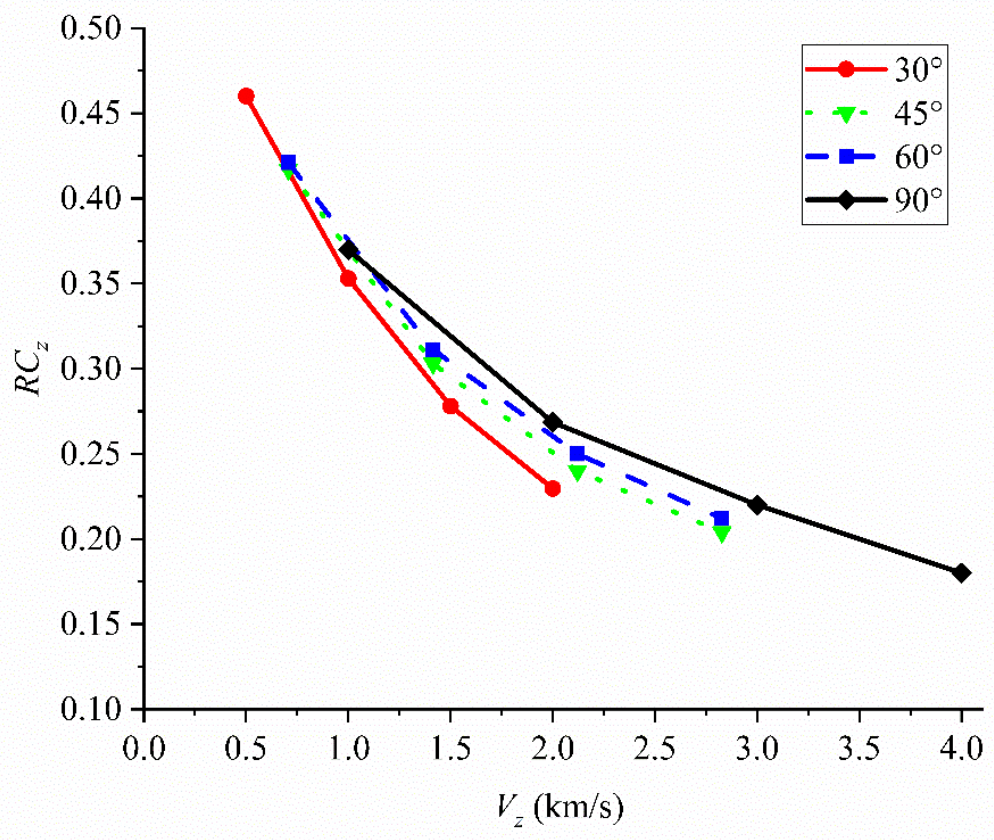

Fig. 8. Coefficient of restitution for the projectile impact along the $z$-direction. 


\subsection{Impact analysis of rotated double-layer graphene sheet}

A rotated double-layer graphene sheet is obtained from $\theta$ anticlockwise rotation for bottom layer graphene. The rotation angle is continuously in the range of $0^{\circ}$ to $30^{\circ}$ owing to the six-fold symmetry [34]. The rotated double-layer graphene sheet with rotation angles of $3,7,10,14,20$, and 27 degrees is impacted by the $\mathrm{C}_{180}$ fullerene with the energy of $100.825 \mathrm{eV}$. Table 1 shows the impact (KE Impact) and rebound (KE $\mathrm{Kebound}_{\text {) }}$ kinetic energies of the projectile under the impact simulation of different rotation angles double-layer graphene sheet. Under the same initial kinetic energy projectile impact, we observe that the larger the rotation angle of the double-layer graphene sheet, the smaller the rebound kinetic energy of the projectile. Kim et al. [29] revealed that the electronic band structure of the rotated double-layer graphene has obvious features compared to the conventional stacked double-layer graphene. Due to the misorientation-angle between the top and bottom graphene sheets, when the graphene sheet is impacted, the graphene sheet with the misorientation-angle will generate more intense vibration, which will restore the structure to conventionally stacked doublelayer graphene $\left(0^{\circ}\right)$.

In our simulation, by measuring the deformation at the center of the graphene sheet and the axial-wave at the edges of the graphene sheet, the double-layer graphene sheet with a high rotated angle has a larger amplitude, which means that the graphene sheet generates more intense vibration. When fullerene impacts the graphene sheet, the graphene sheet with a high rotated angle generates more intense vibration, and the kinetic energy of fullerene is more effectively converted into the deformation energy of the graphene sheet. This implies that the high rotation angle graphene sheet has a better energy absorption for the projectile. 
Table 1. The kinetic energy of the projectile during impact with a rotated double-layer graphene sheet

\begin{tabular}{llllllll}
\hline$\theta\left(^{\circ}\right)$ & 0 & 3 & 7 & 10 & 14 & 20 & 27 \\
\hline $\mathrm{KE}_{\text {Impact }}(\mathrm{EV})$ & 100.825 & 100.825 & 100.825 & 100.825 & 100.825 & 100.825 & 100.825 \\
$\mathrm{KE}_{\text {Rebound }}(\mathrm{EV})$ & 5.034 & 4.940 & 4.893 & 4.876 & 4.860 & 4.830 & 4.820 \\
\hline
\end{tabular}

\section{Conclusions}

In this work, we performed a comprehensive study on the stress wave propagation, coefficient of restitution, and energy absorption of graphene sheets under the impact of $\mathrm{C}_{180}$ fullerene projectile using $\mathrm{MD}$ simulations. The cone-wave and axial-wave velocities were calculated to be $4.14 \mathrm{~km} / \mathrm{s}$ and $18.18 \mathrm{~km} / \mathrm{s}$, respectively. High velocity cone-wave and axial-wave propagations can better dissipate the kinetic energy of the projectile. These properties are responsible for the higher impact protection performances of graphene sheets. Furthermore, based on the computed values of the coefficient of restitution for the double-layer graphene sheet, our MD simulation results show that the double-layer graphene sheet has a better energy reduction effect in the vertical direction for low angle and high velocity incident. Subsequently, the impact simulation of a rotated double-layer graphene sheet shows that a high rotation angle graphene sheet has a better energy absorption for the projectile. Overall, the results presented in this paper show that single-layer and double-layer graphene sheets have huge potentials for future applications as impact protection materials.

\section{Acknowledgments}

This work was supported in part by NSFC under Grant No. 11902159, Natural Science Foundation of Jiangsu Province under Grant No. BK20170820, Swansea University New Faculty Grant, China Scholarship Council, the State Administration of Foreign 
Experts Affairs under Grant No. G20190010023, the Open Foundation of the Suzhou

Smart City Research Institute and Suzhou University of Science and Technology under Grant No. SZSCR2019010.

\section{References}

[1] Bunch JS, van der Zande AM, Verbridge SS, Frank IW, Tanenbaum DM, Parpia JM, et al. Electromechanical resonators from graphene sheets. Science. 2007;315(5811):490-3.

[2] Hecht DS, Hu L, Irvin G. Emerging transparent electrodes based on thin films of carbon nanotubes, graphene, and metallic nanostructures. Advanced Materials. 2011;23(13):1482-513.

[3] Liu Y, Dong X, Chen P. Biological and chemical sensors based on graphene materials. Chemical Society Reviews. 2012;41(6):2283-307.

[4] Changgu L, Xiaoding W, Kysar JW, James H. Measurement of the elastic properties and intrinsic strength of monolayer graphene. Science. 2008;321(5887):385-8.

[5] Yan C, Lee PS. Stretchable Energy Storage and Conversion Devices. Small. 2014;10(17):3443-60.

[6] Sadeghzadeh S. Nanoparticle mass detection by single and multilayer graphene sheets: Theory and simulations. Applied Mathematical Modelling. 2016;40(17-18):7862-79.

[7] Xue C, Gao H, Hu Y, Hu G. Hyperelastic characteristics of graphene natural rubber composites and reinforcement and toughening mechanisms at multi-scale. Composite Structures. 2019;228.

[8] Bohm S. Graphene against corrosion. Nature Nanotechnology. 2014;9(10):741-2.

[9] Young RJ, Kinloch IA, Gong L, Novoselov KS. The mechanics of graphene nanocomposites: A review. Composites Science and Technology. 2012;72(12):1459-76.

[10] Mao HY, Laurent S, Chen W, Akhavan O, Imani M, Ashkarran AA, et al. Graphene: promises, facts, opportunities, and challenges in nanomedicine. Chemical Reviews. 2013;113(5):3407-24.

[11] Arao Y, Mizuno Y, Araki K, Kubouchi M. Mass production of high-aspect-ratio fewlayer-graphene by high-speed laminar flow. Carbon. 2016;102:330-8.

[12] Castro Neto AH, Guinea F, Peres NMR, Novoselov KS, Geim AK. The electronic properties of graphene. Reviews of Modern Physics. 2009;81(1):109-62.

[13] A.Balandin A, Ghosh S, Bao W, Calizo I. Superior Thermal Conductivity of SingleLayer Graphene NANO LETTERS. 2008;8(3):902-7.

[14] Palermo V, Kinloch IA, Ligi S, Pugno NM. Nanoscale Mechanics of Graphene and Graphene Oxide in Composites: A Scientific and Technological Perspective. Advanced Materials. 2016;28(29):6232-8.

[15] Khalili SMR, Haghbin A. Investigation on design parameters of single-walled carbon nanotube reinforced nanocomposites under impact loads. Composite Structures. 2013;98:253-60.

[16] Rahman AS, Mathur V, Asmatulu R. Effect of nanoclay and graphene inclusions on the low-velocity impact resistance of Kevlar-epoxy laminated composites. Composite Structures. 2018;187:481-8. 
[17] Wei X, Meng Z, Ruiz L, Xia W, Lee C, Kysar JW, et al. Recoverable Slippage Mechanism in Multilayer Graphene Leads to Repeatable Energy Dissipation. ACS Nano. 2016;10(2):1820-8.

[18] Lee J-H, Veysset D, Nelson K, Thomas E. Laser induced projectile impact test (LIPIT): A micron-scale ballistic test for high-strain rate mechanical study of nano-structures. Aps Meeting. 2012:26008.

[19] Lee JH, Loya PE, Lou J, Thomas EL. Materials science. Dynamic mechanical behavior of multilayer graphene via supersonic projectile penetration. Science. 2014;346(6213):1092-6.

[20] Natsuki T, Natsuki J. Transverse impact analysis of double-layered graphene sheets on an elastic foundation. International Journal of Engineering Science. 2018;124:41-8.

[21] Natsuki T, Ni Q-Q. Theoretical analysis of transverse impact response in double layer graphene sheets. AIP Advances. 2016;6(1).

[22] Xia K, Zhan H, Hu D, Gu Y. Failure mechanism of monolayer graphene under hypervelocity impact of spherical projectile. Scientific Reports. 2016;6:33139.

[23] Sadeghzadeh S. Computational design of graphene sheets for withstanding the impact of ultrafast projectiles. Journal of Molecular Graphics and Modelling. 2016;70:196-211.

[24] Kawai T, Okada S, Miyamoto Y, Oshiyama A. Carbon three-dimensional architecture formed by intersectional collision of graphene patches. Physical Review B. 2005;72(3).

[25] Bai Z, Zhang L, Liu L. Bombarding Graphene with Oxygen Ions: Combining Effects of Incident Angle and Ion Energy To Control Defect Generation. The Journal of Physical Chemistry C. 2015;119(47):26793-802.

[26] Plimpton S. Fast Parallel Algorithms for Short-Range Molecular Dynamics. Journal of Computational Physics. 1995;117(1):1-19.

[27] Humphrey W, Dalke A, Schulten K. VMD: Visual molecular dynamics. Journal of Molecular Graphics. 1996;14(1):33-8.

[28] Stuart SJ, Tutein AB, Harrison JA. A reactive potential for hydrocarbons with intermolecular interactions. The Journal of Chemical Physics. 2000;112(14):6472-86.

[29] Kim K, Coh S, Tan LZ, Regan W, Yuk JM, Chatterjee E, et al. Raman spectroscopy study of rotated double-layer graphene: misorientation-angle dependence of electronic structure. Physical Review Letters. 2012;108(24):246103.

[30] Jain SK, Juričić V, Barkema GT. Structure of twisted and buckled bilayer graphene. 2D Materials. 2016;4(1).

[31] Sockalingam S, Gillespie JW, Keefe M. Dynamic modeling of Kevlar KM2 single fiber subjected to transverse impact. International Journal of Solids and Structures. 2015;6768:297-310.

[32] Haque BZ, Chowdhury SC, Gillespie JW. Molecular simulations of stress wave propagation and perforation of graphene sheets under transverse impact. Carbon. 2016;102:126-40.

[33] Xu J, Sun Y, Wang B, Li Y, Xiang Y, Chen X. Molecular dynamics simulation of impact response of buckyballs. Mechanics Research Communications. 2013;49:8-12.

[34] Hou J, Deng B, Zhu H, Lan Y, Shi Y, De S, et al. Magic auxeticity angle of graphene. Carbon. 2019;149:350-4. 